\title{
Developing the Biomolecular Screening Facility at the EPFL into the Chemical Biology Screening Platform for Switzerland
}

\author{
Gerardo Turcatti ${ }^{*}$
}

Biomolecular Screening Facility (BSF), Ecole Polytechnique Fédérale de Lausanne (EPFL), Lausanne, Switzerland

\begin{abstract}
The Biomolecular Screening Facility (BSF) is a multidisciplinary laboratory created in 2006 at the École Polytechnique Fédérale de Lausanne (EPFL) to perform medium and high throughput screening in life sciences-related projects. The BSF was conceived and developed to meet the needs of a wide range of researchers, without privileging a particular biological discipline or therapeutic area. The facility has the necessary infrastructure, multidisciplinary expertise and flexibility to perform large screening programs using small interfering RNAs (siRNAs) and chemical collections in the areas of chemical biology, systems biology and drug discovery. In the framework of the National Centres of Competence in Research (NCCR) Chemical Biology, the BSF is hosting 'ACCESS', the Academic Chemical Screening Platform of Switzerland that provides the scientific community with chemical diversity, screening facilities and know-how in chemical genetics. In addition, the BSF started its own applied research axes that are driven by innovation in thematic areas related to preclinical drug discovery and discovery of bioactive probes.
\end{abstract}

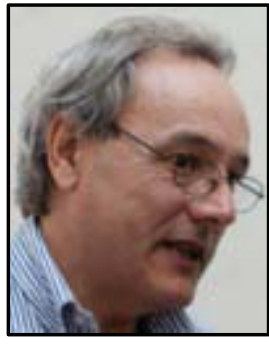

Gerardo Turcatti

Keywords: Academic screening, assay automation, assay development, high-content screening, hits prioritization, hits-toleads, phenoytpic assays, target-based assays.

\section{INTRODUCTION}

Until recently, screening for bioactive molecules was almost exclusively carried out by the pharmaceutical industry; however, at the beginning of the $21^{\text {st }}$ century the first academic programs were initiated [1]. It is now widely recognized that academic screening centers play an important role in drug and biological probe discovery, thanks to the valuable multidisciplinary contributions of academic researchers. Over the last decade, many universities and research units worldwide have integrated screening and early drug discovery disciplines to create screening centers or facilities following different models according to their institutional mandates.

Planned during 2005 and started in the spring of 2006 at the Life Sciences Faculty of the Ecole Polytechnique Fédérale de Lausanne (EPFL), the Biomolecular Screening Facility (BSF) was conceived with a long-term vision, structured plan, and operating model that was ambitious and original in an academic setting at that time. The three main criteria in the creation of the BSF are described as follows:

\section{Fulfilling Needs}

The platform was designed and constructed according to the needs and ideas of researchers from different disciplines, with various life science applications and objectives in mind. The platform does not privilege any particular biological discipline or therapeutic area and performs screens in (but

*Address correspondence to this author at the Biomolecular Screening Facility, Ecole Polytechnique Fédérale de Lausanne (EPFL), Station 15, Lausanne 1015, Switzerland; Tel: +41 2169396 66;

E-mail: gerardo.turcatti@epfl.ch not limited to) the areas of cancer research [2, 3], neurobiology $[4,5]$, and metabolic and infectious diseases [6] for researchers interested in drug discovery, chemical biology and systems biology. This 'generic' and flexible concept was extended to the type of screening assays and the nature of compounds to be tested. The BSF performs a large variety of assays in 96 and 384 well plate format for in vitro biochemical target-based and cellular assays, including image-based phenotypic assays (High Content Screening [HCS]). Regarding the compounds that are screened, our researchers objectives prompted us to select and acquire libraries of synthetic chemicals, natural products and synthetic small interfering RNA duplexes, (siRNAs) for gene knockdown screens up to the genome scale.

\section{Experienced Staff}

We hired technicians and scientists with previous pharmaceutical or biotechnological industrial experience in order to avoid the time-consuming learning process for establishing industrial practices in compound management, assay validation, automation and statistical data analysis. Moreover, for a broader coverage of different disciplines with a small team, we selected platform members with multidisciplinary skills. Thanks to the professional level and commitment of the staff, the BSF has been able to handle a large variety of projects and deliver the first results from screening campaigns from early in its activities, while continuing to construct and develop the platform infrastructure $[2,4]$.

\section{Applied Research at the Screening Platform}

To perform screening in an academic setting, it was essential to innovate by developing an independent applied 
research program. The earliest example of this was the development of an in-house system for information and data management (LIMS) that was planned from the creation of the BSF. Our customized LIMS gives us important advantages in terms of complete control of the system, quality of screening operations, and provides us with operating cost savings, as there is no need to pay license fees for third-party software.

The BSF handles incoming project proposals according to a precise management workflow (Fig. 1) that enables collaborative interaction between the platform staff and the external project members. In addition to our fundamental activities, the BSF also has an educational role, and the research axes of the platform seek to innovate the screening process through new approaches and technologies. It is our aim, and we believe our mission as academics, to contribute to the development of future technologies and methodologies in this evolving field through selected, specifically funded, research projects.

\section{PROGRAMMED DEVELOPMENT OF THE SCREENING PLATFORM}

The BSF mission is to perform chemical and siRNAs screens and to have open access [7] for training users and for participating in educational programs at the EPFL School of Life Sciences. The platform was designed to fulfill the specific needs of researchers and was constructed in two phases during the period 2006-2008; today the center occupies about $230 \mathrm{~m}^{2}$. One of the major challenges during the initial phase was to design an integrated robotic workstation that was flexible enough to perform all the major automated tasks in the lab, from traditional compound management and in vitro screening experiments to whole genome siRNA transfections/cellular screens. During the second phase, a second workstation was designed to increase the screening capacity, in particular for in vitro target-based, chemical screens. Recently, an integrated platform based around a non-contact acoustic dispenser was acquired, completing the high-end fluidic handling equipment.

After the platform was validated by delivering results from screening campaigns for local EPFL researchers and the associated community, we extended our activities through interaction with national networks, initiatives and programs. In 2007 the BSF platform was integrated in the Swiss Initiative for Systems Biology [8], allowing us to specialize in large-scale gene knock-down screens using whole genome siRNAs collections, principally through informative screening by imaging assays [9-11]. More recently, the BSF is currently hosting 'ACCESS', an academic chemical screening platform for Switzerland, in the framework of the National Centre of Competences in Research (NCCR)-Chemical Biology, led by the University of Geneva and the EPFL [12].

\section{The NCCR for Chemical Biology}

The main mission of the NCCR 'Chemical Biology Visualization and Control of Biological Processes Using

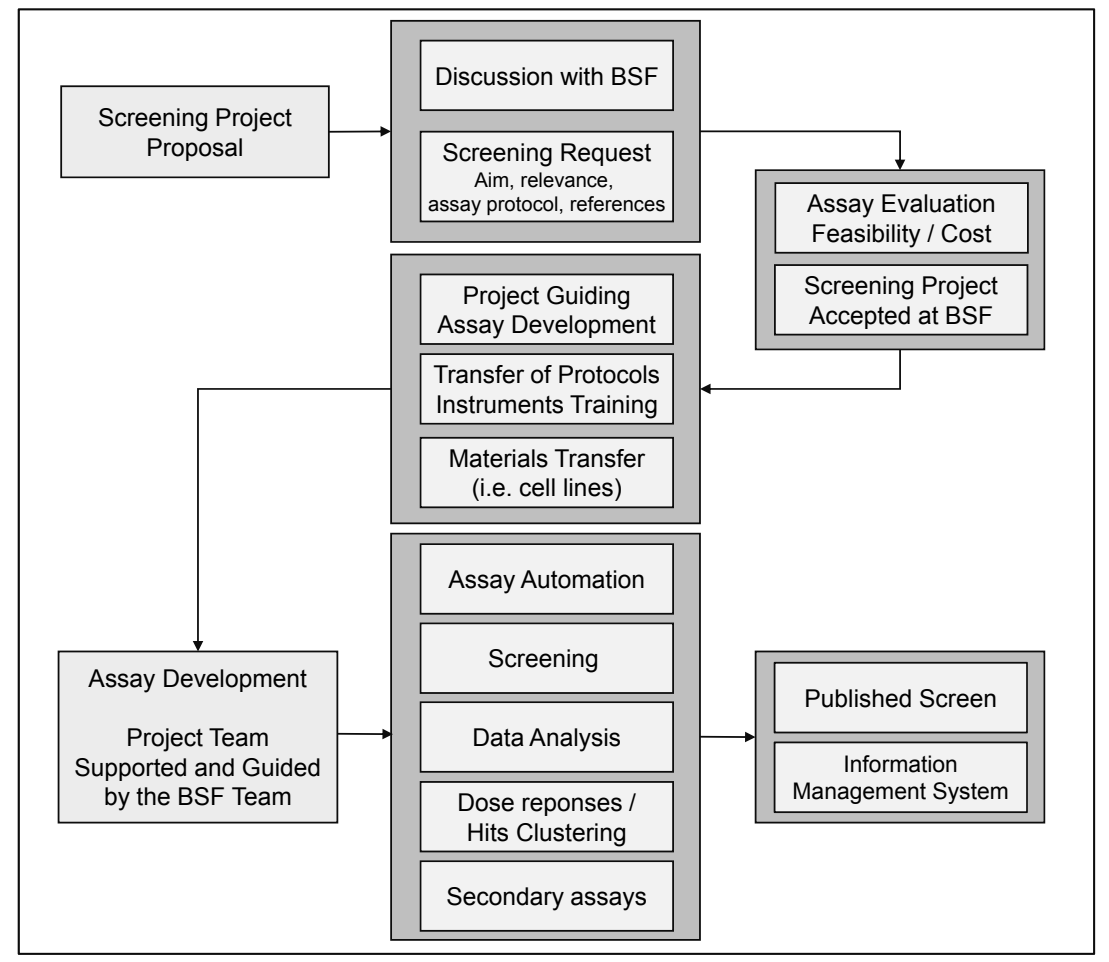

Fig. (1). Screening projects workflow at the screening platform BSF/ACCESS. Project proposal submissions are followed by a series of planned steps, where the initial evaluation of users questions and needs is critical for defining the type of interaction and support provided by the BSF. The initial part of the collaboration between the project and BSF teams is essential for a smooth transfer of knowledge and tools between the two groups, and is invaluable for successful assay validation and subsequent screening operations. Consulting, advising, educating and training are among the most time consuming and valued tasks of the screening platform team. Additional BSF support to our partners includes assistance for grant requests and co-application for funding collaborative programs. 
Chemistry' is to use chemical tools to obtain a better understanding of life at the molecular level [13]. Until now, few technologies could characterize the countless biochemical activities that constitute a living cell in detail. In the NCCR Chemical Biology, chemists, biochemists, physicists and cell biologists are developing innovative techniques based on small molecules and proteins to obtain new information about cellular processes and to control them in situ. These new tools will be applicable to various biological phenomena, such as visualizing the activity of selected proteins during cell division and investigating how the cell membrane controls protein activity. The NCCR is also engaged in establishing a platform for academic chemical screening in Switzerland (ACCESS), which aims to develop a new generation of bioactive molecules.

\section{ACCESS}

One of the goals of this national project is to establish a platform that provides the Swiss scientific community with access to chemical diversity, screening facilities and knowhow in chemical genetics. The ACCESS platform, centralized at the EPFL-BSF, is fast becoming a focal point of the NCCR and will enable the scientific community in Switzerland to benefit from the enormous possibilities offered by chemical biology.

The BSF actively participated in the preparation of this NCCR project, in particular in the elaboration of the plan to further develop the existing platform into a national specialized center for chemical screening. The main objectives and developments carried out during the first three years (2011-2013) of this national project are described in Table 1.

\section{The BSF-ACCESS Chemical Collection Approaches 100 '000 Compounds}

Thanks to its important infrastructure, the BSF has been performing screens since 2006 and handling collections of up to 130 '000 siRNAs and $65^{\prime} 000$ chemical compounds. About $15^{\prime} 000$ of these small molecules are available for screening by our whole community of researchers. The remaining collections (50'000 compounds, mostly GPCRs and kinase-targeted libraries) are available for projects with specific partnering agreements between the research groups and the suppliers of these focused chemical libraries. The BSF 'generic set' of 15 ' 440 chemicals that we make available to every screening project is essentially composed of the Hit-Finder collection (from Maybridge) of 14'400 compounds representing drug-like diversity and the NINDS II collection (from Microsource Discovery Systems) of 1'040 bioactive compounds. To provide our users with access to a larger chemical diversity, we expanded the existing BSF collections by designing, selecting and purchasing the sets and sub-collections described in Table $\mathbf{2}$.

The first ACCESS collection (the Chemically Diverse set) was designed and selected using an innovative approach that led to the selection of about 54'000 commercially available compounds covering the known chemical space. 'Escape from flatland' [14] has been suggested as being beneficial for clinical success of compounds in the drug discovery pipeline.

Although molecular shape is recognized as an important property in drug discovery, often little attention is paid to this feature during the creation of compound collections for screening [15]. Using visualization tools developed inhouse, we applied an innovative molecular shape-based approach for the analysis of chemical space in the design of the ACCESS screening collection (Fig. 2). In addition to the shape criteria, the classic and widely accepted descriptors (essentially those included in the rule of five) were introduced to evaluate whether they yield a more pharmacologically relevant chemical space. The design of the 'Diverse Screening Collection for Chemical Biology', that included molecular shape as a descriptor, resulted in an enrichment of three-dimensional molecules. This set is composed of nearly 54'000 compounds in 8'611 clusters, yielding an average redundancy of six compounds per cluster.

\section{Collecting Compounds from Academic Laboratories and Establishing Compound Management Procedures}

Another goal of ACCESS is to create a screening library containing chemical compounds collected from Swiss academic labs that will be stored and managed at the EPFL. The strategy for collecting these compounds has been defined and pilot tests have been performed with the members of the NCCR that donated the first sets of compounds. We validated our strategic approach for collecting these unique synthetic molecules after establishing standard operating procedures and guidelines for proper handling, transfer and tracking of donated compounds. The diagram shown in Fig. (3) briefly summarizes these procedures, including some aspects of the global compound management strategy.

\title{
Table 1. NCCR-Chemical Biology-ACCESS: Goals and Current Status of Developments
}

\author{
CHEMICAL LIBRARIES \\ - Building the ACCESS chemical collection: Increasing the available chemical diversity at the BSF; currently about 95 '000 small molecules \\ - The Swiss chemical collection: A strategy for collecting compounds from academic synthetic chemistry labs has been developed and validated \\ INSTRUMENTAL INFRASTRUCTURE \\ - Compound management and quality control: Definition and validation of the compound management instrumental infrastructure and \\ standard operating procedures for handling and controlling the chemical integrity of chemicals \\ - $\quad$ ACCESS screening outstation: An ACCESS antenna has been opened at the University of Geneva with selected screening instrumentation \\ including an automated screening microscope allowing faster progression of in house HCS projects \\ ENLARGING THE COMMUNITY OF BSF COLLABORATORS THROUGH ACCESS \\ - Various chemical screens have been performed for the NCCR-Chem Biol partners and pilot projects are progressing towards lead optimization \\ - Opening the platform to Swiss academic and non profit-research institutions
}


Table 2. The BSF/ACCESS Chemical Collections Stored at the BSF (October 2013)

\begin{tabular}{|l|c|c|l|}
\hline \multicolumn{1}{|c|}{ Name of the Set } & Number of Compounds & Suppliers & Comments and Status \\
\hline \hline The Chemically diverse set & $54^{\prime} 000$ & $\begin{array}{c}\text { Enamine Chemdiv } \\
\text { Life Chemicals }\end{array}$ & $\begin{array}{l}\text { Commercially available chemical space covered with an average } \\
\text { redundancy of six compounds per cluster. The collection was } \\
\text { designed using shape as one of the chemical descriptors resulting } \\
\text { in a diverse collection enriched with 3D structures and sp3 centers. }\end{array}$ \\
\hline $\begin{array}{l}\text { Protein-protein interaction } \\
\text { (PPi) }\end{array}$ & $5^{\prime} 441$ & Life Chemicals & $\begin{array}{l}\text { This set is composed of compounds chosen by PPi-Machine- } \\
\text { Learning-Method (895) and PPi-Rule-of-four (4546). }\end{array}$ \\
\hline Natural products (NPs) & 2 '654 & $\begin{array}{l}\text { Analyticon, } \\
\text { InterBioscreen }\end{array}$ & $\begin{array}{l}\text { These compounds are purified organic molecules from fractionated } \\
\text { extracts of two sources, plants and bacteria. }\end{array}$ \\
\hline $\begin{array}{l}\text { The Prestwick Chemical } \\
\text { Library (PCL) }\end{array}$ & $1^{\prime 2} 280$ & $\begin{array}{l}\text { Prestwick Chemicals } \\
\text { A set of known bioactive molecules, approved drugs. }\end{array}$ \\
\hline Kinases inhibitors & 192 & Selleckchem & This selected set is composed of 192 kinases inhibitors. \\
\hline $\begin{array}{l}\text { The natural products- } \\
\text { inspired set }\end{array}$ & About 15'000 & $\begin{array}{l}\text { Currently in progress. Design and selection of a collection of } \\
\text { synthetic molecules using all commercially available NPs and } \\
\text { derivatives as reference. }\end{array}$ \\
\hline
\end{tabular}

\section{A Successful Pilot Screening Campaign}

One of the first screening campaigns performed in the context of the NCCR Chemical Biology has been used by ACCESS to validate the new instruments, new chemical libraries and procedures (Fig. 4). The primary assay for this screen was an in vitro enzymatic assay that used a homogenous time-resolved fluorescence (HTRF) read-out and aimed to discover inhibitors of the enzyme Sepiapterin Reductase (SPR). The rational approach from the team of Professor Kai Johnsson was based on previous research showing that blocking the activity of SPR affects the cellular levels of the molecule tetrahydrobiopterin (BH4), which is critical for the production of neurotransmitters such as serotonin and dopamine. $\mathrm{BH} 4$ deficiency causes similar neurological problems to the side effects of sulphonamide drugs. Thanks to the initial ACCESS screening of the collection of known drugs (The Prestwick Chemical Library) and subsequent validations, the group of Professor Johnsson at the EPFL showed for the first time that sulphonamides bind to the enzyme that synthesizes BH4. Thus, the group's work showed that sulphonamides interfere with the biosynthesis of neurotransmitters, which can account for their neurological side effects. It also helps us understand how the activity of these drugs relates to their molecular structure, and

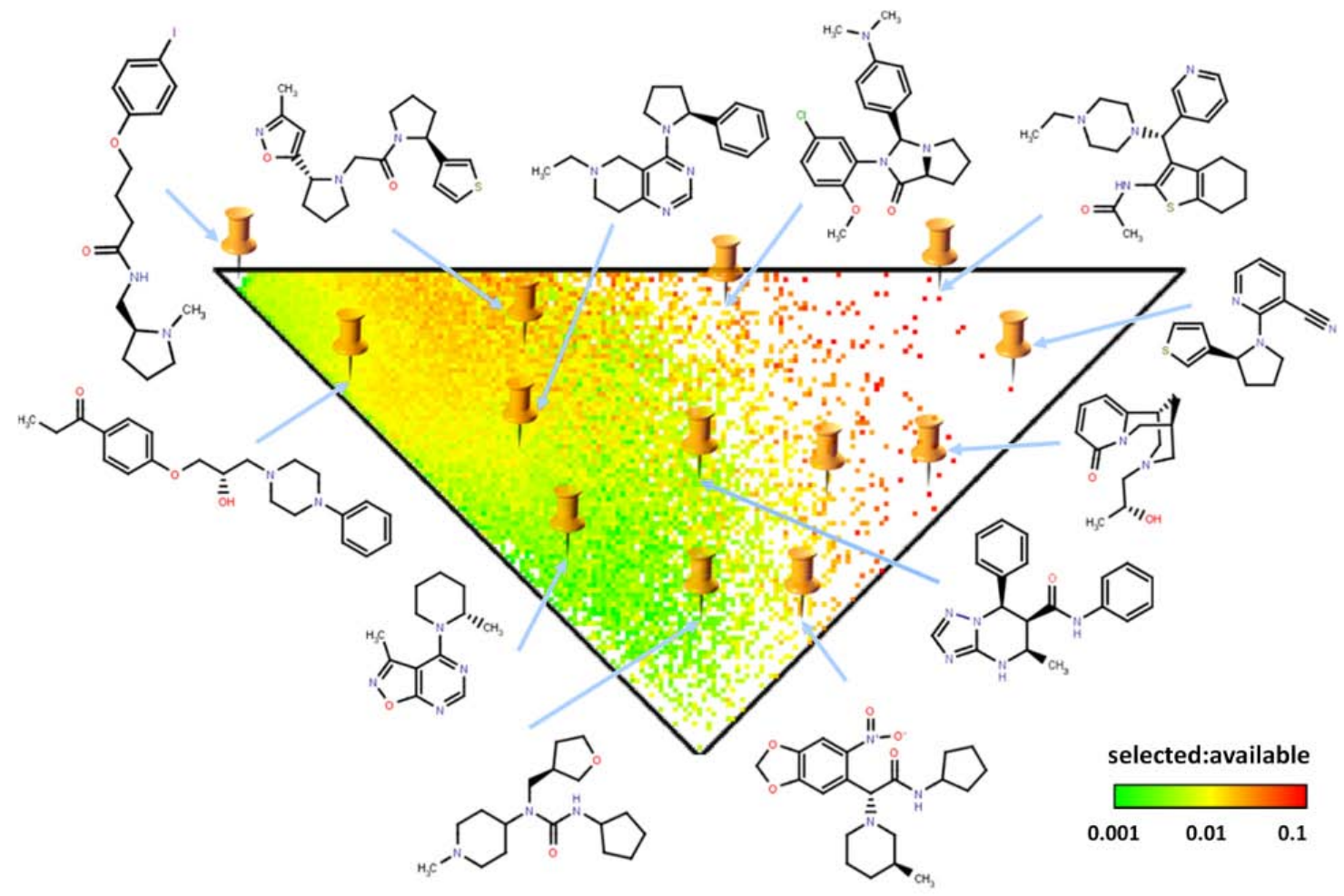

Fig. (2). Density map of the 54'000 compounds in the ACCESS subset 'chemical diverse collection'. The design of a diverse screening collection for chemical biology including molecular shape as a descriptor resulted in an enrichment of three-dimensional molecules. 


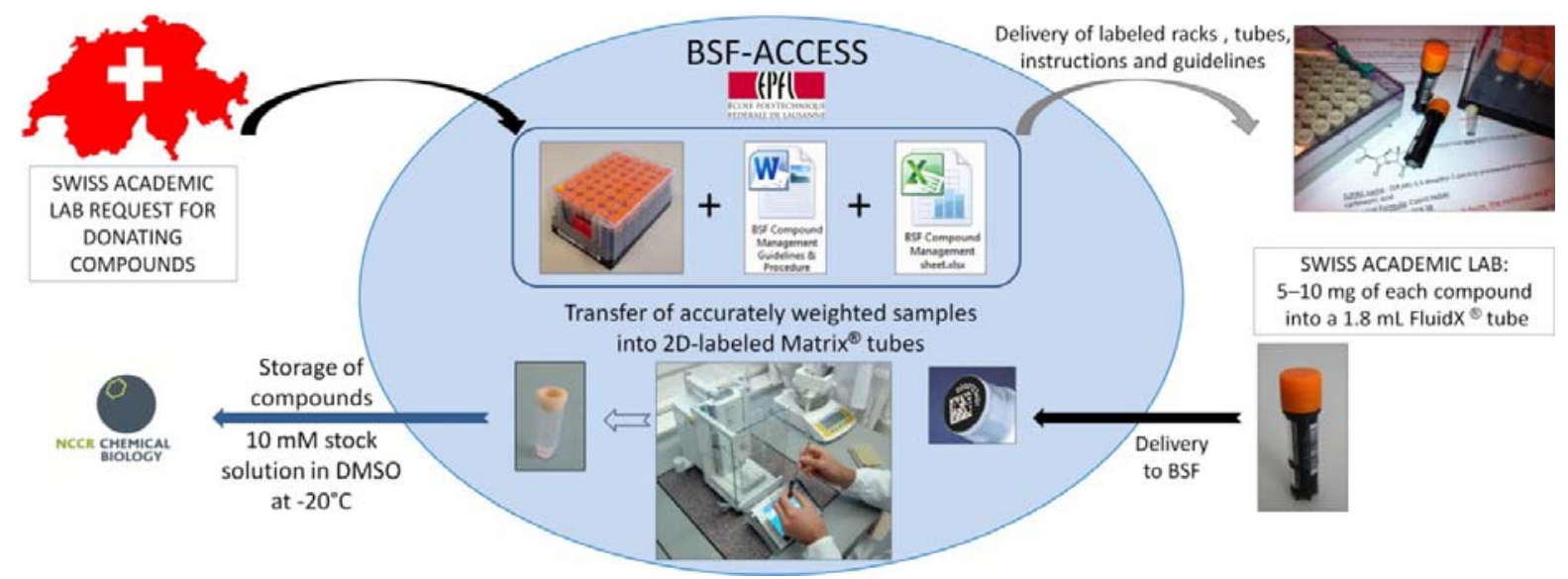

Fig. (3). Overall process for collecting synthetic compounds from academic groups.

suggests ways in which their clinical use could be optimized [16].

This project, which was the first to use the ACCESS diverse collection of 54'000 compounds, is currently validating the expanded chemical structures from validated hits using secondary cellular assays and is progressing towards valuable leads. The outcome of this and other screening campaigns performed so far has been a useful assessment of the value and quality of the collections for the discovery of new bioactive chemical entities in chemical biology and drug discovery projects.

\section{Opening the Screening Platform to Swiss Researchers}

In its current phase the ACCESS-BSF platform is opening its access to other Swiss Academic Institutions and the NCCR-Chemical Biology has made a call for high impact chemical screening proposals within Switzerland.
After these proposals are evaluated by the ACCESS steering committee, the management board of the NCCR will sponsor the first selected projects to enter the platform. The selection procedure will be based on chemical biology, scientific relevance, and feasibility of adapting the proposed validated biochemical or biological assay to a high throughputscreening format.

\section{Financial Aspects}

The platform's instruments have been mainly financed by the EPFL, with important contributions by NCCR Chemical Biology and SystemsX.ch. The pricing of most screens is based on moderate fees for services and consumables, with a contribution to instrument maintenance and repair. Some projects are carried out in a collaborative framework through specific grants that may also cover part of the staffing costs. It is our policy to privilege academic projects and industrial

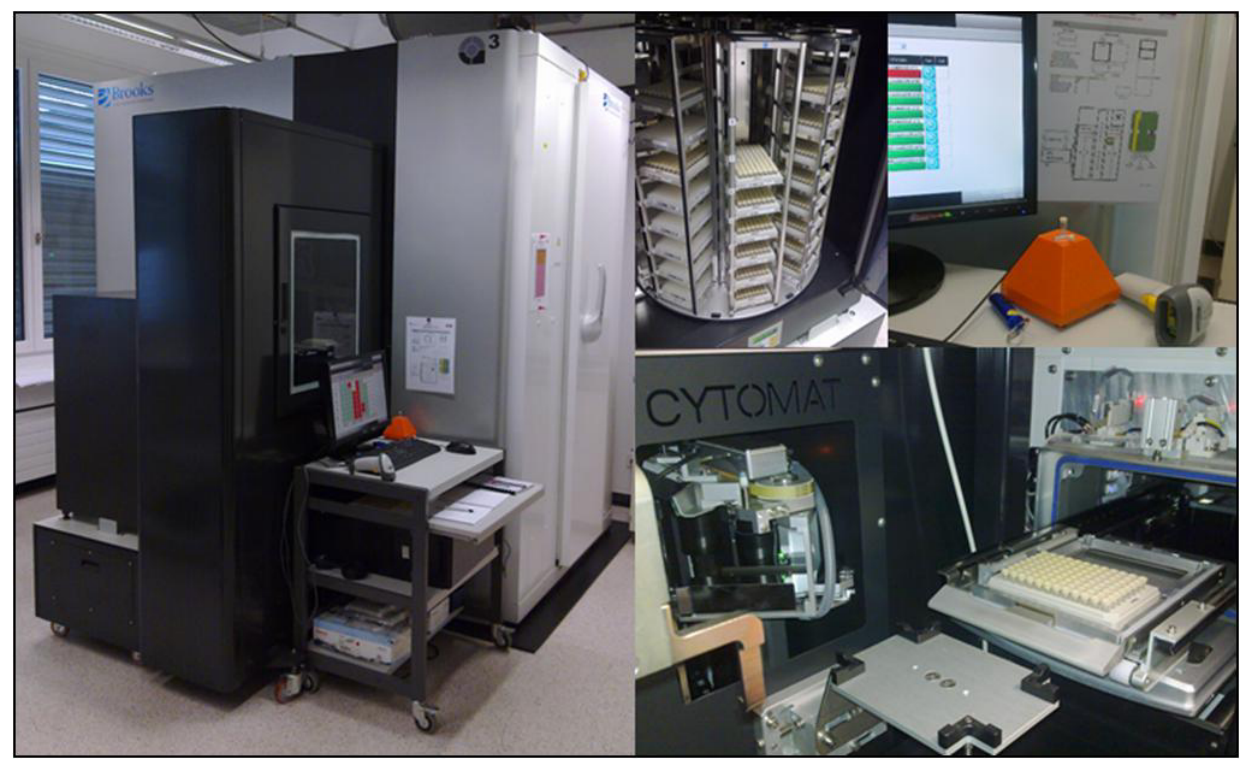

Fig. (4A). Compound management using an automated storage system. Compounds dissolved at a concentration of $10 \mathrm{mM}$ in dimethyl sulfoxide in $2 \mathrm{D}$-bar coded capped tubes $(300 \mathrm{uL})$ are stored at $-20^{\circ} \mathrm{C}$ in an automated store in a controlled low humidity environment. Plated compounds are also stored in the system using sealed 1D coded 384 well plates. Compound tracking and inventory is performed by a Laboratory Information Management System (LIMS) developed by the BSF. 


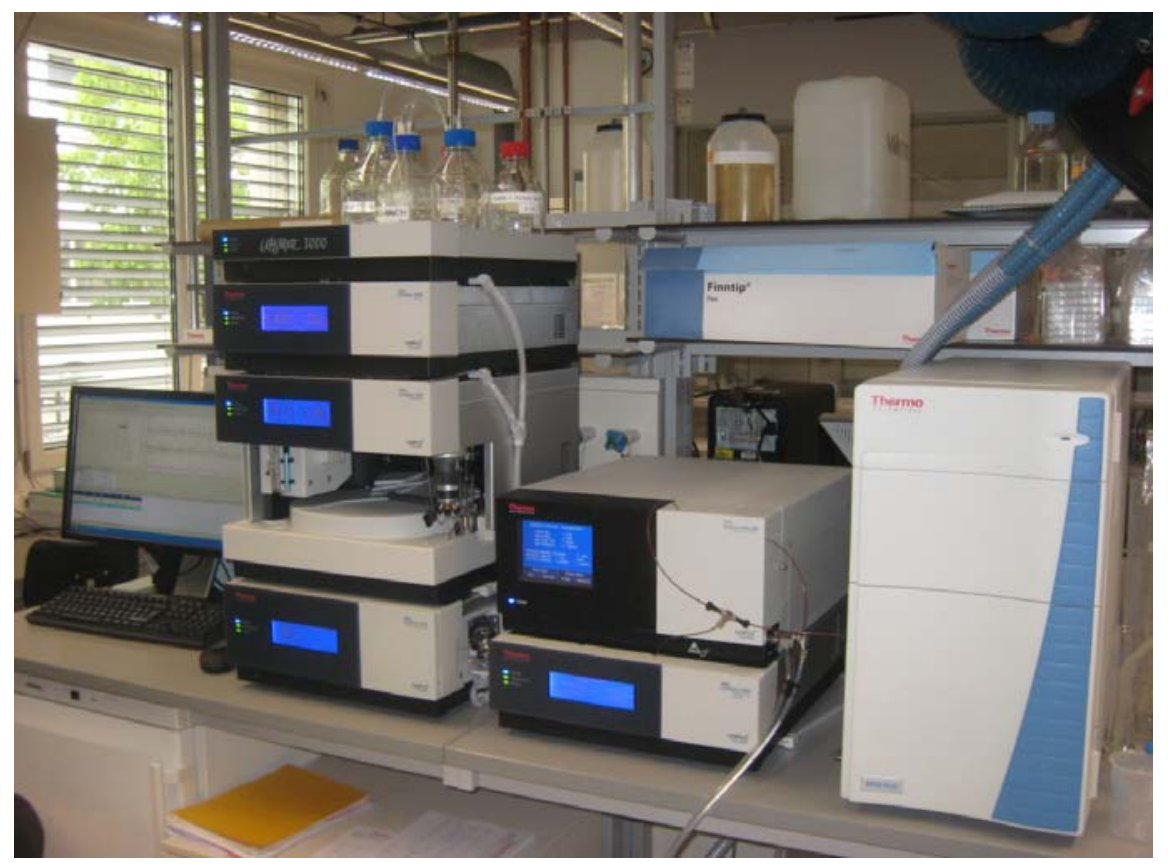

Fig. (4B). Checking the chemical integrity of chemical compounds using an UHPLC-MS system. To estimate the purity of the whole collection within a reasonable time frame, an ultra high performance liquid chromatography system connected to different detectors (Mass spectrometer, UV and CAD) was installed and the workflow from data acquisition to data processing has been validated. Hits from screens will be systematically analyzed and it is planned to randomly analyze the whole ACCESS collections according to the throughput.

collaborations. So far, services for private companies have been limited to access to some instruments (i.e. automated microscopes).

\section{BSF Applied Research and Industrial Collaborations}

Contribution to the development of new screening technologies and approaches is, in our opinion, an important mission for screening platforms in an academic environment. Indeed, academic screening centers are in an ideal position to understand the diverse needs of a large community of researchers and to identify topics for further investigation that could result in innovative tools and approaches that advance the discovery process. We strongly believe that screening and drug discovery can only be integrated as academic disciplines through active independent research, and this is where screening facilities, such as the BSF, have a major role to play.

Based on our interpretation of the current needs of the field and our own areas of scientific expertise, we selected the research axes of the BSF to design informative and relevant assays and to explore the chemical diversity provided by natural sources.

Few of the projects in which the BSF has been actively involved were purely academic collaborations. One early example of an academic project was the development and validation of a new high throughput screening (HTS) assay for direct unbiased monitoring of telomerase activity without post amplification of the resulting DNA products (TeloSpot) [2]. Later on, in another academic collaboration involving three partners, we used cyanobacteria extracts to screen telomerase inhibitors; after fractionation of active extracts, this led to the identification of two sulfoquinovosyl diacylglycerol active structures that were further validated after chemical synthesis [3].

\section{Industrial Collaborations}

Most of the research projects involving or led by the BSF are collaborations with industrial partners, mainly through joint industry-academia projects granted by the Swiss Commission for Technology and Innovation (CTI). This is illustrated by the two examples described below.

In 2010, we started a four partner, CTI-funded, collaborative project between Prof. Radtke (EPFL), Dr. Turcatti (BSF-EPFL), and the Swiss companies Debiopharm and Atheris. This project aimed to identify novel peptides that interfere with pathways controlling cell fate as potential therapeutic compounds to fight cancer stem cells [17]. During this collaboration, the EPFL performed screens using an innovative co-culture cellular assay and in vitro assays using Atheris venoms. After fractionation, these yielded validated candidates that are currently being optimized by the Atheris 'Reverse-Discovery' lead optimization platform in collaboration with Debiopharm [18] This project illustrates the added value of the multi-partner collaborative approach and for the BSF represents the second example of the exploration of products from natural sources; in this case, peptides with therapeutic potential present in the Atheris venoms collections [19].

Regarding HCS research, the BSF is leading a two year, CTI-funded collaboration with LyncéeTec S.A. [20] (an EPFL spin off company that develops instruments for Digital Holographic Microscopy [DHM]). In the context of this collaboration, we have validated screening applications after automating a DHM microscope and optimizing this 
technology that enables label-free quantitative cellular imaging in cell viability screens [21]. This technique appears to be superior to other label-free interferometric microscopy for quantitative cellular analysis, and is unique in its suitability for HTS and time-lapse label-free imaging [22]. We are currently developing a series of other validated applications, such as screening by imaging of the GABA-A ligand-gated chloride channel receptor [23], profiling compounds against cancer cell lines for anti-mitotic and anti-proliferative activity, and cellular assays in physiologically-relevant 3D matrices.

\section{FUTURE PROSPECTS}

The BSF is evolving into a specialized national centre for chemical biology that aims to advance selected projects to the drug discovery phase. One of the future challenges will be to achieve this evolution without detrimental effects on less applied areas of research. Our goal is to maintain our flexibility to satisfy the diverse needs of academic researchers, while simultaneously increasing the number of projects aimed at discovery of new chemical probes. Moreover, we believe that rather than being incompatible, the general and specialized functions of our centre complement each other. Opening the platform as a national centre significantly increased our activities, therefore our limited manpower could result in delays during busy times; however, thanks to the NCCR Chemical Biology, two more positions have been created, increasing the number of full time employees to 6.6. Depending on the success and evolution of the centre, further increases in manpower could be envisioned. With a few exceptions, staff members are employed on a temporary basis, with contracts renewed annually up to a maximum of four years, thus, as is the case with many academic technological platforms, there is the additional challenge of maintaining the high level of expertise and know-how acquired over the years.

The BSF has some exciting perspectives in view, and we foresee a promising future for academic screening in general. As our ambitious goals are put into practice, it is possible that some plans may be modified or adapted. This may require flexibility and support from our institution, in particular concerning the status and perception of technological platforms that have evolved to become more than just services providers. In this respect, we think that the research projects started in our laboratories merit attention for their significant contributions in the multiple domains that involve screening, in particular in early drug discovery. For this to continue, researchers should have access to academic grants for projects closely related to life sciences that target technologies and methodologies, and not only for basic biological research. Stronger links between academic screening centres and collaborative research efforts are the perfect opportunity to make major advances in discovery technologies and methodological approaches.

\section{CONFLICT OF INTEREST}

The author confirms that the manuscript has no conflict of interest.

\section{ACKNOWLEDGEMENTS}

Funding: School of Life Sciences, EPFL, Swiss National Science foundation - NCCR Chemical Biology and SystemsX.ch for significant contributions allowing the development of the core facility.

CTI research program (grant No. 10917.1 PFLS-LS): 'Venomics for discovering new leads for cancer therapy'. Bridge Industry Project (BIP -SystemsX.ch): 'High content screening by digital holographic imaging'. CTI research program (grant No. 12669.1 PFLS-LS): 'A digital holographic microscope for label-free high content screening'.

Special thanks to the Biomolecular Screening Facility staff; Nathalie Ballanfat, Damiano Banfi, Julien Bortoli, Billy Breton, Marc Chambon, Gérald Cruciani, Antoine Gibelin and Ruud van Deursen. CTI research program (grant No. 12669.1 PFLS-LS): Benjamin Rappaz, Pascal Jourdain and Sandra Borel.

\section{REFERENCES}

[1] Stein, R. L. High-throughput screening in academia: the Harvard experience. J. Biomol. Screen., 2003, 8 (6), 615-619.

[2] Cristofari, G.; Reichenbach, P.; Regamey, P. O.; Banfi, D.; Chambon, M.; Turcatti, G.; Lingner, J. Low- to high-throughput analysis of telomerase modulators with Telospot. Nat. Methods, 2007, 4 (10), 851-853.

[3] Makhlouf Brahmi, M.; Portmann, C.; D'Ambrosio, D.; Woods, T. M.; Banfi, D.; Reichenbach, P.; Da Silva, L.; Baudat, E.; Turcatti, G.; Lingner, J.; Gademann, K. Telomerase inhibitors from cyanobacteria: isolation and synthesis of sulfoquinovosyl diacylglycerols from Microcystis aeruguinosa PCC 7806. Chemistry - A European Journal, 2013, 19 (14), 4596-4601.

[4] Ouertatani-Sakouhi, H.; El-Turk, F.; Fauvet, B.; Roger, T.; Le Roy, D.; Karpinar, D. P.; Leng, L.; Bucala, R.; Zweckstetter, M.; Calandra, T.; Lashuel, H. A. A new class of isothiocyanate-based irreversible inhibitors of macrophage migration inhibitory factor Biochemistry, 2009, 48(41), 9858-9870.

[5] Ouertatani-Sakouhi, H.; El-Turk, F.; Fauvet, B.; Cho, M.-K.; Pinar Karpinar, D.; Le Roy, D.; Dewor, M.; Roger, T.; Bernhagen, J.; Calandra, T.; Zweckstetter, M.; Lashuel, H. A. Identification and characterization of novel classes of macrophage migration inhibitory factor (MIF) inhibitors with distinct mechanisms of action. J. Biol. Chem., 2010, 285(34), 26581-26598.

[6] Magnet, S.; Hartkoorn, R. C.; Székely, R.; Pató, J.; Triccas, J. A.; Schneider, P.; Szántai-Kis, C.; Orfi, L.; Chambon, M.; Banfi, D.; Bueno, M.; Turcatti, G.; Kéri, G.; Cole, S. T. Leads for antitubercular compounds from kinase inhibitor library screens. Tuberculosis, 2010, 90 (6), 354-360.

[7] The Biomolecular Screening Facility. http://bsf.epfl.ch/. (Accessed October 30, 2013)

[8] SystemsX.ch. The swiss initiative in systems biology. http://www.systemsx.ch/. (Accessed October 30, 2013).

[9] Snijder, B.; Sacher, R.; Ramo, P.; Liberali, P.; Mench, K.; Wolfrum, N.; Burleigh, L.; Scott, C. C.; Verheije, M. H.; Mercer, J.; Moese, S.; Heger, T.; Theusner, K.; Jurgeit, A.; Lamparter, D.; Balistreri, G.; Schelhaas, M.; De Haan, C. A. M.; Marjomaki, V.; Hyypia, T.; Rottier, P. J. M.; Sodeik, B.; Marsh, M.; Gruenberg, J.; Amara, A.; Greber, U.; Helenius, A.; Pelkmans, L. Single-cell analysis of population context advances RNAi screening at multiple levels. Mol. Syst. Biol., 2012, 8, 579.

[10] Balestra, Fernando R.; Strnad, P.; Flückiger, I.; Gönczy, P. Discovering Regulators of centriole biogenesis through siRNAbased functional genomics in human cells. Dev. Cell, 2013, 25 (6), 555-571.

[11] Moreau, D.; Scott, C.; Gruenberg, J. A novel strategy to identify drugs that interfere with endosomal lipids. Chimia. (Aarau), 2011, 65 (11), 846-848. 
[12] Swiss National Centre of Competences in Research-Chemical Biology. http://www.nccr-chembio.ch/. (Accessed October 30, 2013).

[13] Sturzenegger, S.; Johnsson, K.; Riezman, H. NCCR Chemical Biology: Interdisciplinary Research Excellence, Outreach, Education, and New Tools for Switzerland. Chimia. (Aarau), 2011, 65 (11), 832-834

[14] Lovering, F.; Bikker, J.; Humblet, C. Escape from flatland: increasing saturation as an approach to improving clinical success. J.Med. Chem., 2009, 52 (21), 6752-6756.

[15] Sauer, W. H. B.; Schwarz, M. K. Molecular Shape diversity of combinatorial libraries: a prerequisite for broad bioactivity $\dagger$. $J$. Chem. Info. Comput. Sci., 2003, 43 (3), 987-1003.

[16] Haruki, H.; Pedersen, M. G.; Gorska, K. I.; Pojer, F.; Johnsson, K. Tetrahydrobiopterin biosynthesis as an off-target of sulfa drugs. Science, 2013, 340 (6135), 987-991.

[17] Debiopharm. Media. Press Releases. Debio 0826. http://www.debio pharm.com/media/press-releases/62-debio-0826/2883-debiopharmand-epfl-sign-research-project-agreement-to-identify-inhibitors-ofsignalling-pathways-controlling-cell-fate-for-cancer-treatment.html (Accessed October 30, 2013).

[18] Debiopharm. Media. Press Releases. Debio 0826. http://www.debiopharm.com/index.php?option=com content\&vie $\mathrm{w}=$ article $\&$ Itemid $=300041 \& \mathrm{id}=3317 . \quad$ (Accessed October 30, 2013).

[19] Atheris Laboratories. http://www.atheris.ch/. (Accessed October $30,2013)$.

[20] Lyncée tec. http://www.lynceetec.com/. (Accessed October 30, 2013).

[21] Kühn, J.; Shaffer, E.; Mena, J.; Breton, B.; Parent, J.; Rappaz, B.; Chambon, M.; Emery, Y.; Magistretti, P.; Depeursinge, C.; Marquet, P.; Turcatti, G. Label-free cytotoxicity screening assay by digital holographic microscopy. Assay Drug Dev. Technol., 2013, 11 (2), 101-107.

[22] Rappaz, B.; Breton, B.; Shaffer, E.; Turcatti, G. Digital Holographic Microscopy: a quantitative label-free microscopy technique for phenotypic screening. Comb. Chem. High Throughput Screen., 2014, 17 (1), 80-88.

[23] Jourdain, P.; Boss, D.; Rappaz, B.; Moratal, C.; Hernandez, M.-C; Depeursinge, C.; Magistretti, P. J.; Marquet, P. Simultaneous optical recording in multiple cells by digital holographic microscopy of chloride current associated to activation of the ligand-gated chloride channel GABA A receptor. PLOS ONE, 2012, 7 (12), e51041. 